\title{
Larval foraging behaviour and competition in Drosophila melanogaster
}

\author{
GLADYS RUIZ-DUBREUIL $\dagger$, BARRIE BURNET* $\ddagger$, KEVIN CONNOLLY $\ddagger \&$ PETER \\ FURNESS $\S$ \\ $\dagger$ Instituto de Ecologia y Evolucion, Universidad Austral, Valdivia, Chile, $\ddagger$ Department of Molecular Biology and \\ Biotechnology, University of Sheffield, Sheffield S10 2UH and \$Department of Psychology, University of Sheffield, \\ Sheffield S10 2TP, U.K.
}

\begin{abstract}
Populations of Drosophila melanogaster derived by bidirectional selection for high (HA) and low (LA) aggregated oviposition behaviour differ significantly in the duration of the larval period and adult size because of differences in the developmental profiles for feeding rate over successive phases of larval growth. Feeding rates of HA larvae are significantly lower than those of LA larvae during the flexible period of growth which precedes attainment of critical mass for pupation. Consequently the HA larvae have a slower mean rate of development. In the fixed postcritical period of development the feeding rates of HA larvae are significantly higher than those of LA larvae. This causes a greater postcritical growth increment and larger adult flies. HA and LA larvae respond adaptively by changing the expression of components of their foraging behaviour depending on whether they are in or out of food. LA larvae exhibit a more flexible pattern of response and are also more successful competitors when food resources are limiting.
\end{abstract}

Keywords: behaviour, competition, Drosophila melanogaster, foraging, larvae.

\section{Introduction}

The preadult stages of many Drosophila species occupy breeding sites in which resources are transient and patchy in distribution. According to Shorrocks (1990) two species exploiting the same resource patches might never exclude one another entirely, despite competition between them, provided the preadult stages of both species have aggregated and independent distributions. Genetic differences in the tendency to aggregated oviposition behaviour between populations, or species, could create precisely these conditions.

Various environmental factors including olfactory cues and differences in the conformation and texture of the substrate are known to influence the choice of oviposition sites, but aggregated oviposition by $D$. melanogaster females is also known to be genetically controlled and to respond to selection (RuizDubreuil \& del Solar, 1986, 1991, 1993). RuizDubreuil et al. (1994) showed that differences in levels of aggregated oviposition over homogeneous sites actually depend on differences in level of aggregation in the spatial dispersal patterns of the females from the selected populations.

${ }^{*}$ Correspondence.

(C) 1996 The Genetical Society of Great Britain.
The intensity of aggregated oviposition affects the pattern of progeny survival when the unit of resource of each patch is small (Ruiz-Dubreuil et al., 1994). Larvae from females with the most highly aggregated oviposition behaviour may overload the carrying capacity of the limited patches or resource units, causing localized food shortage and an increase in intraspecific competition. The outcome of competition may crucially depend on larval foraging behaviour as well as on the ways in which resources are distributed in the environment.

Almost the entire period of larval development of D. melanogaster is devoted to foraging. Indeed larvae might be described as little feeding machines. The principal components of foraging behaviour have been described by Green et al. (1983). When larvae feed they rake rhythmically over the substrate with the mouth hooks and ingest by means of the pumping action of the muscular pharynx. Individual differences in larval feeding rate are genetically determined and affect larval growth rate, survival and competitive ability (Sewell et al., 1975; Burnet $e t$ al., 1977).

Sokolowski (1980) identified a behavioural polymorphism in $D$. melanogaster larvae. Larvae designated rover have long foraging path lengths, whereas those designated sitter have short path lengths. This 
difference in expression of behaviour was found to be controlled by a major gene foraging (for) located on the second chromosome (De Belle et al., 1989).

The components of larval behaviour are also affected by environmental factors. Green et al. (1983) observed that the rate of larval locomotion changes in the absence of food or in the presence of aversive concentrations of ethanol, which is a metabolic by-product of yeast fermentation in many of the natural breeding sites exploited by $D$. melanogaster (Muhammad-Ali \& Burnet, 1995). Such changes in the rates of performance of behaviours are known as kineses and are discussed in detail by Fraenkel \& Gunn (1940) and Schöne (1984). Here we make a developmental comparison of the behavioural components of larval foraging in populations of $D$. melanogaster selected for differing intensities of aggregated oviposition behaviour and examine whether differences in larval behaviour affect the outcome of competition between them.

\section{Materials and methods}

\section{Stocks}

The stocks of $D$. melanogaster used were high aggregation (HA) and low aggregation (LA) lines, produced by continuous mass selection for more than 360 generations for differences in oviposition behaviour (Ruiz-Dubreuil \& del Solar, 1986, 1991).

Genetically marked stocks LA spa and HA spa were synthesized by incorporating the mutant allele sparkling located on the fourth chromosome into the two selected lines LA and HA. Inversion-containing balancer chromosomes were used in a multigeneration crossing scheme which recovered homozygotes for the first, second and third chromosomes of each respective selected line in association with the spamarker. Serial backcrossing and reisolation of the derived spa- marked HA and LA genotypes each with its parental selected line was then made for several generations using large numbers to prevent bottleneck inbreeding effects. Quality control tests detected no significant differences between the selected lines and the corresponding genetically derived marked lines with respect to aggregated oviposition and larval behaviour.

\section{Synchronized larvae}

Fertilized females aged 3-4 days were allowed to oviposit for $3 \mathrm{~h}$ on a layer of baker's yeast. Eggs were incubated at $25^{\circ} \mathrm{C}$ and newly hatched larvae maintained on fresh yeast at low density to exclude competition for food. Specified larval ages are reckoned in hours after hatching from the egg.

\section{Behavioural observations}

Using a stereomicroscope, each larva was observed in a $9 \mathrm{~cm}$ Petri dish containing a layer of agar gel. The substrate conditions used were: (i) agar gel, 3 per cent; (ii) agar gel overlaid with Drosophila Ringer's solution (Roberts, 1986); and (iii) agar gel overlaid with a suspension of fresh baker's yeast at 48 per cent w/v. A settling down period of 2 min was allowed after transfer to the observation dish before scoring began.

Comparisons of behaviour were made at several points in development $(4,28,52$ and $76 \mathrm{~h})$ using independent random samples of 50 larvae for each age and condition. Repeated measures were made on a random sample of 21 second instar larvae. The method of analysis used for the ensuing paired comparison is described by Sokal \& Rohlf (1981).

Behavioural observations were recorded and analysed using a 10 channel event recorder based on a Toshiba T1200 Laptop computer. The data consisted of a list of successive key presses and releases timed from the start of an observation period. The temporal position of each behaviour is thus stored and the duration of each bout of behaviour can be derived. All the behaviours (see Fig. 1) except feeding were analysed as previously described (Sewell et al., 1975; Green et al., 1983). Feeding was recorded by depressing a specified key for each feeding movement of the mouth hooks. The intervals between successive feeding movements were compiled as a frequency distribution, the form of which was approximately normal for larvae feeding under control conditions on yeast. Breaks between bouts of continuous feeding frequently occurred when the larva was observed under conditions of food withdrawal. These breaks skew the distribution by producing a protracted tail of increasing break lengths. Examination of the frequency distributions of the larvae feeding on yeast indicates that at least 95 per cent of the intervals were less than $1.5 \mathrm{~s}$. This period was therefore used as a threshold and any interval greater than $1.5 \mathrm{~s}$ was defined as an interbout break. The data for feeding rate were then analysed to yield measures of the mean bout lengths and mean break lengths over the whole observation period, together with a mean within-bout rate. The withinbout rate per min measures feeding rate when the effects of breaks have been discounted. 


\section{a}
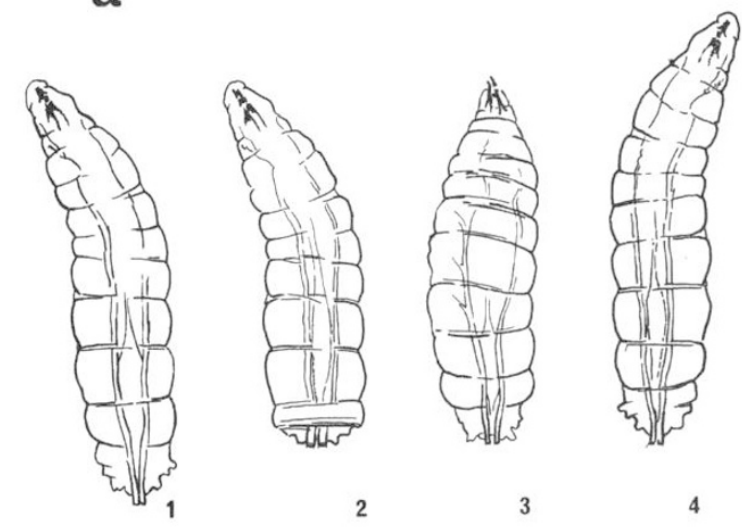

3

4
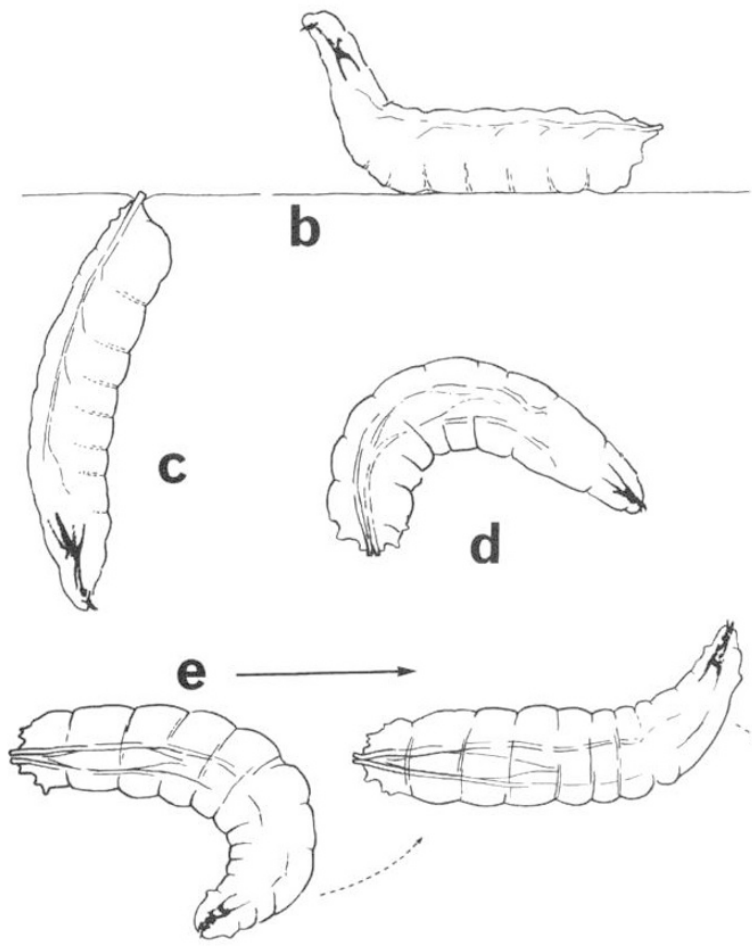

Fig. 1 Larval behaviours of Drosophila melanogaster. (a) Starting from rest in the extended state (1) locomotion proceeds by pulling up the rear end anteriorily (2) creating a wave of compression and extension which passes serially through the segments (3) until it reaches the head (4); a further sequence is then initiated. The sequence (1) to (4) represents a single unit of locomotion. (b) Rearing, (c) digging, (d) turning, (e) bending consists of movement of the head to right or left while scraping with the mouth hooks. Bending is distinct from turning and is specifically associated with feeding. (Redrawn from Green et al., 1983.)

\section{Resource distribution and survival}

Survival rates (Table 1) were measured by placing newly hatched first instar larvae in groups of 50 or 100 into $75 \times 25 \mathrm{~mm}$ diameter glass culture vials containing $5 \mathrm{~mL}$ of standard oatmeal-agar-treacle
Table 1 Survival rate and adult body size in HA and LA strains of Drosophila melanogaster at different larval crowding densities on live yeast medium

\begin{tabular}{lcccc}
\hline $\begin{array}{l}\text { Crowding density } \\
\text { per vial }\end{array}$ & $\begin{array}{l}\text { Survival } \\
\text { to adult }\end{array}$ & $P$ & Body size & $P$ \\
\hline 50 & & & & \\
Strain & & & & \\
HA & $70.9 \pm 1.31$ & 0.3 & $53.5 \pm 0.18$ & $<0.01$ \\
LA & $69.4 \pm 3.88$ & & $51.9 \pm 0.19$ & \\
100 & & & & \\
Strain & & & $52.4 \pm 0.17$ & $<0.01$ \\
HA & $65.2 \pm 3.30$ & 0.9 & $50.3 \pm 0.20$ & \\
LA & $65.9 \pm 3.71$ & &
\end{tabular}

Survival rate is expressed as the mean $\pm S E$ (percentages transformed to angles) for males and females combined. Body size is expressed as the mean $\pm \mathrm{SE}$ of thorax length in arbitrary units (females).

$P$ is the probability that differences between HA and LA strains are the result of chance.

medium seeded with live yeast. Thorax length of adult females was measured as the distance from the pronotum to the distal end of the scutellum. This measure is highly correlated with body weight (Robertson, 1960).

Small glass culture vials, $23 \times 18 \mathrm{~mm}$ diameter, containing Ohba killed yeast medium were used for comparisons of survival (Table 3). Details of this medium are given by del Solar et al. (1976). Ten replicates were used for each condition.

\section{Patch size and distribution}

Glass Petri dishes (14 cm diameter) containing 2 per cent agar gel were used. Live yeast was placed on each plate as follows: $200 \mathrm{mg}$ spread uniformly (UU), $100 \mathrm{mg}$ spread uniformly (UR), $100 \mathrm{mg}$ in a single central patch (P1), $100 \mathrm{mg}$ in three patches (P3), $100 \mathrm{mg}$ in five patches (P5). Patches were each approximately $1 \mathrm{~cm}$ diameter spaced $5 \mathrm{~cm}$ apart. One hundred newly hatched first instar larvae (50 $\mathrm{HA}+50$ LA) were inoculated onto the central patch. Viabilities of the two genotypes were not analysed separately. Fifteen replicate dishes were used for each condition.

\section{Linear environment}

Glass tubes, $2.5 \mathrm{~cm}$ diameter, with $1 \mathrm{~cm}$ diameter access ports spaced at $20 \mathrm{~cm}$ intervals were used to examine the effects of a linear patch distribution. 
The tubes, fixed horizontally, were half filled with a 2 per cent agar gel. Five patches $(1 \mathrm{~cm}$ diameter $)$ each containing $20 \mathrm{mg}$ yeast were then placed $20 \mathrm{~cm}$ apart along the middle of the gel. The ports were lightly bunged with cotton wool. One hundred newly hatched first instar larvae were placed on the central patch. Four replicate tubes were used for each type of monoculture and eight tubes for mixed cultures. Hatched adults were removed each day by aspirating via the access ports.

All experiments were carried out at $25^{\circ} \mathrm{C}$ under standardized conditions of humidity and illumination.

\section{Results}

Developmental comparison of the behavioural components of foraging

Behaviour on food Figure 2 shows components of foraging behaviour for larvae in yeast suspension. The locomotor activity of LA larvae was lower than for HA larvae but locomotion in both populations increased between first and second instar (Fig. 2a). Locomotion by HA larvae remained at a high level into the third instar, then there was a reduction towards the end of the period of larval development.
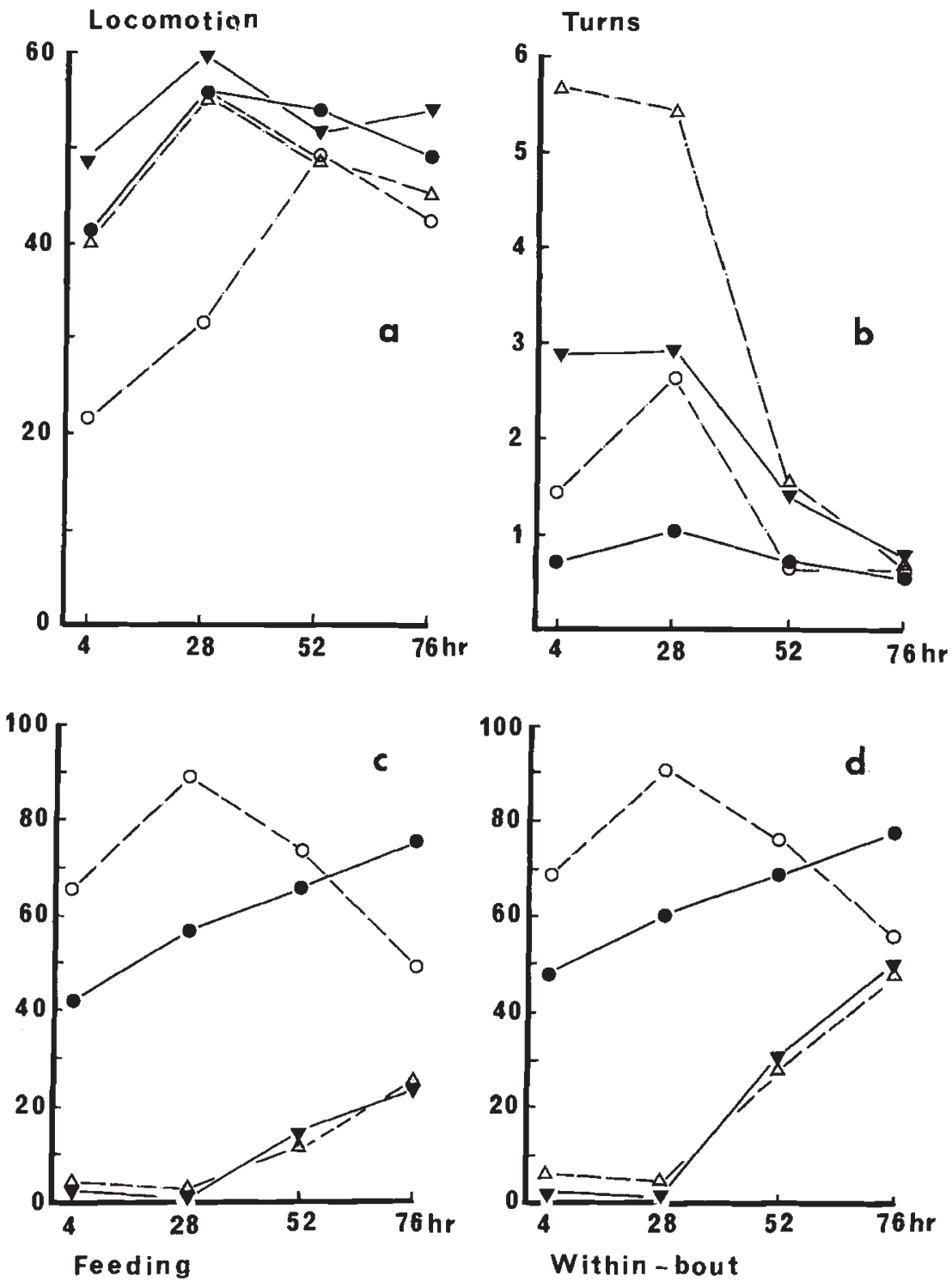

Fig. 2 Components of larval foraging behaviour of Drosophila melanogaster at different ages measured as the mean number of events per min (ordinates) on yeast and on agar. (a) Locomotor activity, (b) turning, (c) feeding rate, (d) within-bout rate of cephalopharyngeal retraction. Circles denote behaviour in yeast, triangles behaviour on agar. Solid symbols denote the HA strain, open symbols the LA strain. 
The locomotor rate of LA larvae progressively increased with age reaching a maximum in the early third instar. The mean locomotor activity of HA larvae is consistently higher at each stage of development and an analysis of variance (ANOVA) indicates significant effects of larval age $\left(F_{3,392}=150, \quad P<0.01\right)$ and strain differences $\left(F_{1,392}=380, \quad P<0.01\right)$. Significant age $\times$ strains interaction $\left(F_{3,392}=46, P<0.01\right)$ confirms that the developmental profiles for locomotion during larval development are different. Larvae attain their critical body mass in early third instar. Around this time locomotor activity of HA and LA larvae is quite similar but, during the important precritical period ( $4 \mathrm{~h}$ and $28 \mathrm{~h}$ ), locomotor activity of HA larvae is significantly higher than it is for LA larvae. The developmental changes in both strains are similar (ANOVA: ages $F_{1,196}=235, \quad P<0.01 ; \quad$ strains $F_{1,196}=673, P<0.01$; interaction $F_{1,196}=3$, NS).

Rates of turning differ significantly with age and also between strains (ANOvA: ages $F_{3,392}=22$, $P<0.01)$; $\quad$ strains $\quad F_{1,392}=20, P<0.01$; interaction $F 3,392=10, P<0.01)$. The HA larvae have a relatively low rate of turning throughout larval development whereas LA larvae turn more frequently $(P<0.01)$ during the precritical period. The rates are similar in the two strains during the third instar (Fig. 2b) and this means that the paths followed by LA larvae during the precritical period of development are more convoluted or meandering than those of the HA larvae.

Continuous periods of feeding activity may be interrupted by intervals in which no feeding occurs. The observed rate of feeding is consequently a result of three variables: bout duration, number of feeding movements within each bout and the duration of intervals between bouts. Differences in feeding rate could arise as an effect of any or all of these. Developmental changes in feeding rates, and within-bout feeding rates, are very similar (Figs. 2c,d), indicating that the gross feeding rate is largely influenced by modulations in the speed at which feeding movements are performed (intrabout rate), rather than by variations of the numbers and durations of feeding bouts.

The developmental profiles for feeding rates of the HA and LA strains are clearly different (ANOVA: ages $F_{3,392}=24, \quad P<0.01 ; \quad$ strains $F_{1,392}=106$, $P<0.01$; interaction $F_{3,392}=245, P<0.01$ ). During the precritical phase of larval development, which precedes attainment of the critical body weight necessary for pupation, the feeding rates of LA larvae were significantly higher than for HA larvae (ANOVA: ages $F_{1,196}=277, \quad P<0.01 ; \quad$ strains $F_{1,196}=605, \quad P<0.01 ; \quad$ interaction $\quad F_{1,196}=16$, $P<0.01)$. The situation in the postcritical period
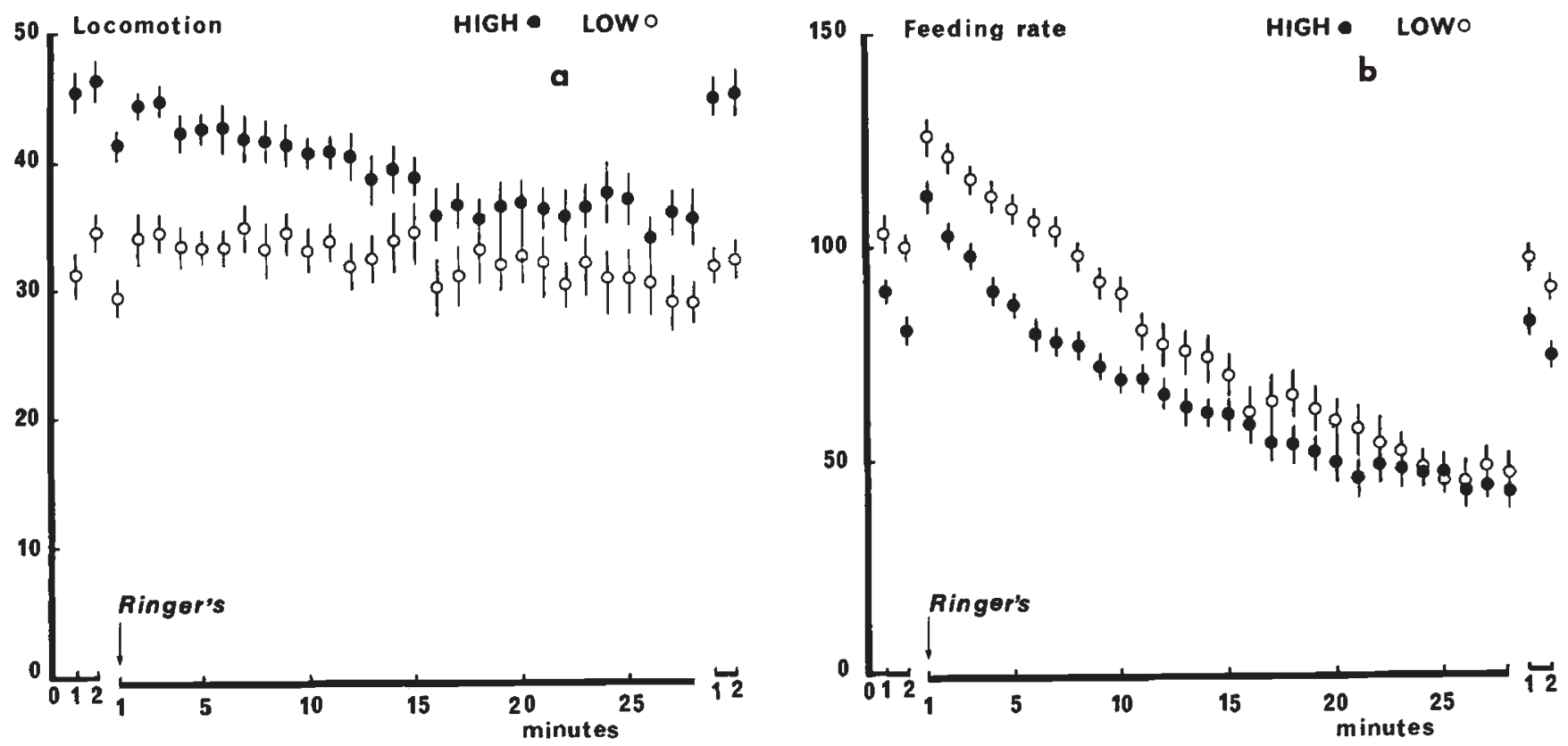

Fig. 3 (a) Locomotion and (b) feeding rate of second instar larvae of Drosophila melanogaster measured in yeast suspension ( $2 \mathrm{~min})$, then in Ringer's solution $(28 \mathrm{~min})$ and again in yeast suspension $(2 \mathrm{~min})$. Symbols denote the mean $\pm \mathrm{SE}$. Solid symbols HA strain, open symbols LA strain. 
(76 h) reversed. Feeding rate for LA larvae in late third instar is significantly below that for the HA larvae $\left(F_{1,98}=210, P<0.01\right)$.

Effects of food withdrawal Food withdrawal was effected by removing larvae from yeast and placing them on the surface of an agar gel. Figure 2a shows that locomotor activity of LA larvae on agar was approximately double compared with that in yeast at $4 \mathrm{~h}$ and $28 \mathrm{~h}$. Locomotion of HA larvae showed only a small increase compared to the relatively high level on food at these ages. In the precritical period turning was significantly higher on agar than yeast in both strains $(P<0.01)$. LA larvae performed the highest rates of turning (Fig. 2b).

The frequencies of feeding movements made by the HA and LA larvae on agar were closely similar (Figs. 2c,d). Young larvae performed very few feeding movements, but the durations of bouts of feeding and the within-bout feeding rates increased during the third instar. The profiles of age related change are virtually identical in the two strains.

Temporal modulations of behaviour On the surface of an agar gel the physical conditions are different from those when a larva is in yeast suspension. Larvae seem to prefer to be partially or wholly immersed in the substrate with the posterior spiracles maintaining contact with the air. Some of the increase in locomotion observed when larvae are moved, from yeast onto agar, may be a response to the reduction of contact, or tactile stimuli, exerted by semi-liquid medium. Feeding movements on agar are made at lower frequency, especially by younger larvae. This is because physical contact, involving immersion of the head, seems to be important for releasing full expression of feeding activity. What is of interest, then, is how larvae respond to attenuation of the supply of nutrients, such as may from time to time occur in a heterogeneous environment. That is, are larvae able to detect dilution of the food supply, and do they respond by adjusting their feeding effort? To examine this question second instar larvae, in their precritical period of growth, were observed in yeast suspension ( $2 \mathrm{~min}$ ), then in Ringer's solution which contained no nutrients $(28 \mathrm{~min})$ and again after being returned to yeast suspension ( $2 \mathrm{~min}$ ). The inclusion of a dual control, by observing behaviour in yeast suspension both before and after the period in Ringer's solution, allows us to test for any persisting physiological effects on behaviour caused by nutrient withdrawal.

After transfer to Ringer's solution a steady decrease in the mean locomotor activity of HA lar- vae took place over a $28 \mathrm{~min}$ period (Fig. 3a), whereas LA larvae showed virtually no change in locomotor activity during the first $15 \mathrm{~min}$ in Ringer's solution. Some reduction subsequently occurred between 15 and $28 \mathrm{~min}$. The mean locomotor activity scores in yeast suspension before and after the Ringer's solution were: $47.6 \pm 1.47$ and $45.2 \pm 1.31$ in the HA strain; $34.6 \pm 1.63$ and $32.1 \pm 1.13$ in the LA strain, respectively. ANOVAs for paired comparisons revealed no significant differences in locomotion in yeast before and after observation in Ringer's solution. Locomotor activity was consistently higher in larvae of the HA strain.

The pattern of response in both strains to attenuation of the nutrient supply was an abrupt increase succeeded by a reduction of feeding rate (Fig. 3). The initial increase in feeding rate seems to indicate that larvae respond by increasing their feeding effort to maintain the rate of food acquisition. The subsequent progressive reduction of the mean feeding rate of both strains was to levels below those in the first yeast control period. The decrement in feeding rate in both strains was caused by a reduction in the mean within-bout rate of cephalopharyngeal retraction and by progressive increase in the mean durations of breaks between feeding bouts. During the first $20 \mathrm{~min}$ in Ringer's solution the feeding rate remained consistently higher in the LA strain, but the two strains tended to converge after $15 \mathrm{~min}$. The control feeding rates in yeast suspension before transfer to Ringer's solution were $90.2 \pm 2.78$ in the HA and $104.9 \pm 4.24$ in the LA strain. After Ringer's solution they were $78.4 \pm 1.82$ in the HA strain and $93.6 \pm 2.82$ in the LA strain. ANOVAs for paired comparisons of feeding rates in yeast revealed that after the period in Ringer's solution they were significantly lower in both strains (HA strain: $F_{1,20}=8.8$, $P<0.01$; LA strain: $\left.F_{1,20}=8.0, P<0.05\right)$. This delay in recovering the initial rates of feeding in the yeast suspension could result from persisting physiological effects which were not expressed in terms of locomotor activity.

\section{Effects of the level and distribution of resources on growth and survival}

'Standard' vial cultures Table 1 compares survival to the adult stage, and adult female body size, for larvae cultured in vials containing standard live yeast medium at optimal (50) and somewhat suboptimal (100) crowding densities (Sang, 1949). No significant strain differences in survival rates were observed at either density, but body sizes of HA females were significantly higher than those of LA females. On 


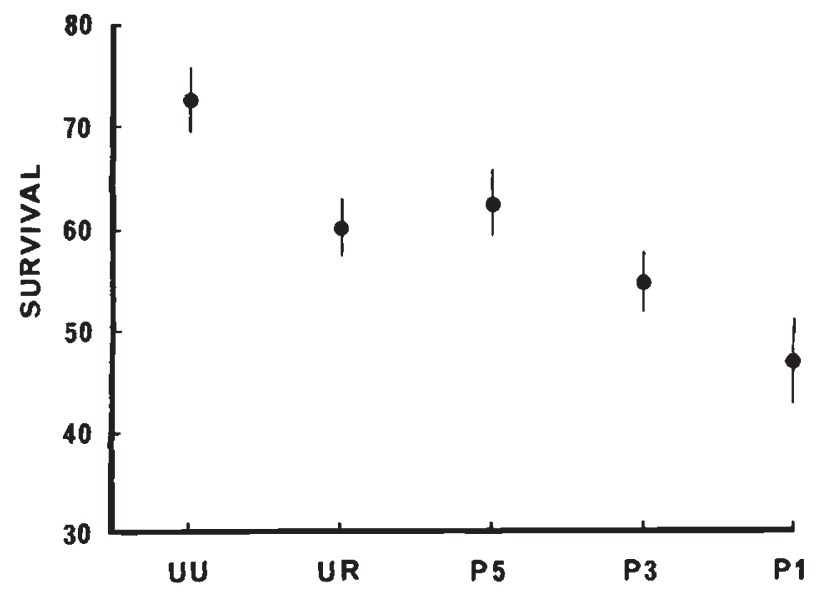

Fig. 4 Effects of the amount of food and the distribution pattern on larval survival of Drosophila melanogaster in large Petri dishes. (UU) $200 \mathrm{mg}$ yeast spread uniformly, (UR) $100 \mathrm{mg}$ yeast spread uniformly, (P1) $100 \mathrm{mg}$ yeast in a single patch, (P3) $100 \mathrm{mg}$ yeast divided into three patches, (P5) $100 \mathrm{mg}$ yeast divided into five patches. Values on the ordinate denote survival (per cent transformed to angles). Means are shown $\pm 2 \times \mathrm{SE}$.

'standard' medium the growth of live yeast is supported by nutrients but, if the larval density is high, overexploitation of the available yeast biomass may occur. Reduced adult body size of both strains at the higher density may reflect a lower available level of food, or possible negative influences of other density-dependent variables (mean body size withinstrains at the two crowding densities is significantly different, $P<0.01$ ).

Patchy resources A larva requires a food provision equivalent to approximately $1 \mathrm{mg}$ of live yeast to develop into a viable adult fly (Burnet et al., 1977). This amount of food gives rise to relatively undersized flies and was chosen as a nonrenewable resource provision to examine the effect of differing modes of spatial distribution on larval survival in large Petri dishes (Fig. 4). Live yeast $(100 \mathrm{mg}$ ) was spread uniformly (UR), or concentrated in one (P1), three (P3), and five (P5) patches. As a further control a nonlimiting quantity of yeast $(200 \mathrm{mg})$ was spread as a uniform layer (UU).

ANOva for survival to adulthood detected significant differences over the whole experiment $\left(F_{5,69}=24, P<0.01\right)$. Survival on the uniform spread (UU) was significantly higher than for each and every distribution pattern at $1 \mathrm{mg}$ of yeast per larva $(P<0.01$ for each comparison). Survival on a single patch $(\mathrm{P} 1)$ was significantly lower $(P<0.01)$ than for any other condition. Survival on five patches (P5) was not significantly different from that on uniform distribution (UR), but significantly higher than on three patches $(P<0.01)$.

Evidently, D. melanogaster larvae forage successfully for a limited food resource which is uniformly distributed, or when it is divided into multiple small patches, but the probability of survival is adversely affected if the same resources are concentrated into few patches.

\section{Competition}

Resources subdivided into a series of patches The relative success of foraging by larvae, expressed in terms of survival and duration of the developmental period, was compared using an extended linear series of patches of food in glass tubes (Table 2). The limiting quantity of food (1 mg per larva) was distributed in equal sized patches spaced $20 \mathrm{~cm}$ apart. Under these conditions the mean rates of larval development, and survival, were significantly higher in the LA strain. In mixed cultures, when LA spa and HA larvae were in competition, survival of LA spa larvae was again significantly higher.

Resources concentrated in a single patch Table 3 shows mean survival at different crowding densities in small vials. At low density there were no significant differences between strains either in monocultures or in mixed cultures, but increasing larval density was attended by a significant reduction in survival within each of the four genotype groups. Not one of the paired comparisons of means within density levels for LA spa versus LA, or HA spa versus $H A$, approached statistical significance, showing that the mutant spa is neutral with respect to survival. ANOVAS showed significant differences in survival between strains at densities of 100 and 200 larvae for both monocultures and mixed cultures. Detailed comparisons of means (Duncan's test) confirmed that survival was consistently and significantly higher for LA over HA genotypes $(P<0.01$ in every case).

\section{Discussion}

The larvae of $D$. melanogaster must reach a critical body weight before they can pupate. The critical weight is reached early in the third instar. At the critical stage larvae have grown to less than half their final achievable body weight. The duration of the precritical period for larval growth can be protracted if nutritional conditions are limiting but, after critical weight is reached, hormonal changes lead to a fixed period of postcritical growth to pupa- 
Table 2 Survival to adult of HA and LA strains of Drosophila melanogaster in glass tubes each containing five resource patches of $20 \mathrm{mg}$ yeast spaced at $20 \mathrm{~cm}$ intervals

\begin{tabular}{lccc}
\hline Strain & HA & LA & $F_{1,6}$ \\
\hline Moncultures & & & \\
Larval period (log $\mathrm{h} \pm \mathrm{SE})$ & $2.1489 \pm 0.0080$ & $2.1217 \pm 0.0070$ & \\
95\% CI (h) & $136-146$ & $128-132$ & \\
Survival & $36.3 \pm 2.6$ & $49.8 \pm 2.4$ & $14.6^{* *}$ \\
\hline Strain & HA & LA spa & $F_{1,14}$ \\
\hline Mixed cultures & & & \\
Survival & $42.3 \pm 2.15$ & $56.7 \pm 1.24$ & $34.0^{* *}$ \\
\hline
\end{tabular}

Survival is expressed as mean \pm SE (angles).

**Denotes differences which are significant at $P<0.01$.

Table 3 Survival to adult in monoculture vials and in mixed culture vials of Drosophila melanogaster containing equal numbers of LA spa and HA (or HA spa and LA) larvae

\begin{tabular}{|c|c|c|c|c|c|}
\hline Monocultures & LA spa & HA & LA & HA spa & $F_{3,36}$ \\
\hline \multicolumn{6}{|l|}{ Larvae } \\
\hline 40 & $80.2 \pm 2.28$ & $83.1 \pm 1.99$ & $82.2 \pm 2.30$ & $80.9 \pm 2.17$ & $0.35 \mathrm{NS}$ \\
\hline 100 & $70.9 \pm 0.65$ & $62.2 \pm 0.94$ & $69.9 \pm 1.21$ & $63.0 \pm 1.09$ & $21.4^{* *}$ \\
\hline 200 & $51.6 \pm 0.64$ & $47.7 \pm 0.51$ & $52.2 \pm 0.82$ & $46.9 \pm 0.61$ & $19.9 * *$ \\
\hline Mixed cultures & \multicolumn{2}{|c|}{ LA spa vs. HA } & \multicolumn{2}{|c|}{ LA vs. HA spa } & $F_{3,36}$ \\
\hline \multicolumn{6}{|l|}{ Larvae } \\
\hline 40 & $81.9 \pm 2.78$ & $81.2 \pm 2.50$ & $79.3 \pm 2.46$ & $80.4 \pm 2.16$ & $0.17 \mathrm{NS}$ \\
\hline 100 & $71.7 \pm 2.46$ & $59.3 \pm 1.10$ & $70.1 \pm 2.33$ & $54.3 \pm 1.99$ & $14.5^{* *}$ \\
\hline 200 & $54.7 \pm 1.31$ & $47.6 \pm 1.32$ & $55.8 \pm 1.27$ & $48.1 \pm 1.63$ & $10.4^{* *}$ \\
\hline
\end{tabular}

Survival is expressed as mean $\pm \mathrm{SE}$ (in angles).

**Denotes differences significant at $P<0.01$ per cent.

tion. The critical weight together with the growth increment in the postcritical period determine the body weight of the adult fly (Bakker, 1961; Robertson, 1963; Burnet et al., 1977).

The developmental profiles for feeding rate of the LA and HA populations show strong genotype-age interaction. Larvae of the LA population have a faster feeding rate in the precritical growth period and reach critical mass earlier than those of the HA population. However, in the fixed postcritical growth period, superior feeding rate in the HA population leads to greater growth increment and superior adult body size. These conclusions are in accord with those of Burnet et al. (1977) for populations which were selected directly for differences in larval feeding rate.

It is interesting that larvae of both populations show an increase in turning rate when removed from food onto agar, the LA larvae showing the greater response, and consequently their foraging paths differ from each other. Also, larvae of the selected populations differ with respect to the effect of nutrient withdrawal on their locomotor activity. For the HA larvae the effects are relatively minor, but during the precritical period of development, LA strain larvae are significantly less actively engaged in locomotion when they are in yeast suspension compared with when they are on agar, and this could be energetically more efficient. Such a difference was not observed between larvae compared in a liquid milieu (i.e. in yeast suspension and then in Ringer's solution). Increased locomotor activity of larvae on agar was previously observed by Green et al. (1983), who proposed that appetitive food seeking behaviour in a non-nutritive environment could be the reason. The results presented here raise the ques-

(C) The Genetical Society of Great Britain, Heredity, 76, 55-64. 
tion of how far thigmotactic stimuli deriving from body contact with the substrate may be confounded with the effects of nutrient availability in comparisons of locomotor and feeding activity in yeast and on agar. Feeding movements on agar were made infrequently by larvae of both selected strains during the precritical period of development. Contact with liquid by the head of the larvae almost always induces feeding activity, but food per se is obviously not the only relevant influence because the effect is the same whether the liquid is yeast suspension, Ringer's solution, or distilled water. The effects of nutrient withdrawal are likely to be manifest in the initial acceleration and subsequent decrement of feeding rate over time shown by both selected strains in Ringer's solution and the rapid recovery to nearly the control rate which occurs when larvae are returned to yeast suspension. From this perspective, nutrient withdrawal in liquid milieu affects feeding activity of larvae of the two selected populations in essentially the same way.

The genetic basis of the divergence in larval behaviours in the HA and LA populations in response to selection may lie in the pleiotropic effects of the genes controlling the aggregated oviposition behaviour of the adult females. Such pleiotropic interactions between larval and adult behaviours involving rover and sitter phenotypes are described by Periera \& Sokolowski (1993). Also, correlated changes in larval characters may be caused indirectly by disruption of the linkage relations of coadapted gene complexes (Falconer, 1989). Bearing in mind the small number of linkage groups in the genome of D. melanogaster, the latter is quite likely.

Concentration of limited food resources into a single available patch is suboptimal for larval survival compared with division of the same absolute amount into several accessible patches. The reasons for this require further investigation and may derive from physical interference between individuals. Contact between larvae frequently leads to temporary cessation of feeding, and when this happens larvae sometimes slash at each other with the mouth hooks. On the other hand, larval interactions with the metabolic products of other individuals can cause both facilitation and interference in growth and survival (Dawood \& Strickberger, 1969; Budnik \& Brncic, 1975).

Graf \& Sokolowski (1989) compared the foraging behaviour of rover and sitter larvae on agar and yeast at different stages in development by measuring their path lengths. They observed only small effects of substrate quality on larvae during the pre- critical period ( 24 and $48 \mathrm{~h}$ ), and greatest expression of the rover phenotype in the postcritical period ( 72 and $96 \mathrm{~h}$ ). Furthermore, food absence reduced the expression of the rover phenotype. Exact comparisons with our findings are precluded by differences in the genotypes and conditions, and because the behavioural measures that have been used in the two studies are not the same. Nevertheless, it seems that, because of the apparently late onset in the postcritical period of full expression of the rover phenotype, the for locus can have only a minor impact on differences in growth to attainment of larval critical weight, but could have an important influence on the postcritical growth increment contributing to adult body size, as well as on larval vagility affecting choice of pupation sites (Sokolowski \& Hansell, 1983). If a larval behaviour is primarily relevant to food acquisition it would be expected that it should be strongly expressed when food is scarce, and that it will be strongly expressed during the precritical phase of development when it will have greatest effect on viability.

From the standpoint of competition, differences in larval behaviour, development rates and adult body size between the HA and LA populations have consequences for immediate and prospective fitness. Robertson (1957) found body size to be positively correlated with egg production. On the other hand recent findings by Santos et al. (1994) show that genetically larger individuals are at a disadvantage when larval density increases. In a patchy environment differences in foraging behaviour should crucially determine the outcome when competition between alternative genotypes actually occurs. The LA larvae are clearly more flexible in adjusting their locomotor rate depending on food availability, and the higher feeding rate of the LA larvae during the precritical growth period leads to a clear prediction that they should be the superior competitors in the short-term. This is amply confirmed by the significantly higher mean survival rate of LA larvae when the food supply is limiting, whether resources are concentrated into a single patch or subdivided into many patches. Our findings demonstrate that larval feeding rate in the precritical period of larval development of $D$. melanogaster is a principal component of fitness for this scramble-type competitor.

\section{Acknowledgements}

This work was carried out under the auspices of the British Council's Academic Links Scheme, with additional support from the Royal Society, Fondecyt grants 1930394 and DID-UACH S-92-43, and an 
equipment grant from the University of Sheffield Research Fund, each of which is gratefully acknowledged. Fig. 1 was redrawn from Green et al. (1983) with permissison of Academic Press Ltd., London.

\section{References}

BAKKER, K. 1961. An analysis of factors which determine success in competition for food among larvae of Drosophila melanogaster. Arch. Neerl. Zool, , 14, 200-281.

BUDNIK, M. AND BRNCIC, D. 1975. Effects of larval biotic residues on viability in four species of Drosophila. Evolution, 29, 777-780.

BURNET, B., SEWELL, D. AND BOS, M. 1977. Genetic analysis of larval feeding behaviour in Drosophila melanogaster. II. Growth relations and competition between selected lines. Genet. Res., 30, 149-161.

DAwood, M. M. AND STRICKBERGER, M. w. 1969. The effects of larval interaction on viability in Drosophila melanogaster. III. Effects of biotic residues. Genetics, $\mathbf{6 3}$, $213-220$.

DE BELLE, J. S., HILIKER, A. J. AND SOKOLOWSK1, M. B. 1989. Genetic localization of foraging (for): a major gene for larval behaviour in Drosophila melanogaster. Genetics, 123, 157-163.

DEl SOlAR, E., GUiJón, A. M. AND WAlker, L. 1974. Choice of coloured substrates for oviposition in Drosophila melanogaster. Boll. Zool., 41, 253-260.

FALCONER, D. S. 1989. Introduction to Quantitative Genetics, 3rd edn. Longman Scientific and Technical, Harlow, Essex.

FRAENKEL, G. S. AND GUNN, D. L. 1940. The Orientation of Animals. Clarendon Press, Oxford.

GRAF, S. A. AND SOKOlowsKI, M. B. 1989. Rover/sitter Drosophila melanogaster larval foraging polymorphism as a function of larval development, food-patch quality and starvation. J. Insect Behav., 2, 301-313.

GREEN, C. H., BURNET, B. AND CONNOLLY, K. J. 1983. Organisation and patterns of inter- and intraspecific variation in the behaviour of Drosophila larvae. Anim. Behav., 31, 282-291.

MUHAMMAD-ALI, A. Z. K. AND BURNET, B. 1995. Ethanol tolerance and variation at the alcohol dehydrogenase (Adh) locus of Drosophila mauritiana. Heredity, 74, $438-444$.

PERIERA, H. S. AND SOKOLOWSKl, M. B. 1993. Mutations in the larval foraging gene affect adult locomotory behavior after feeding in Drosophila melanogaster. Proc. Natl. Acad. Sci. U.S.A., 90, 5044-5046.

ROBERTs, D. в 1986. Drosophila: a Practical Approach. IRL
Press, Oxford.

Robertson, F. W. 1957. Studies in quantitative inheritance. XI. Genetic and environmental correlation between body size and egg production in Drosophila melanogaster. J. Genet., 55, 428-443.

ROBERTSON, F. W. 1960. The ecological genetics of growth in Drosophila. 1. Body size and developmental time on different diets. Genet. Res., 1, 288-304.

ROBERTSON, F. W. 1963. The ecological genetics of growth in Drosophila. 6. The genetic correlation between the duration of the larval period and body size in relation to larval diet. Genet. Res., 4, 74-92.

RUiZ-Dubreuil, D. G. AND DEL SOLAR, E. 1986. Effect of selection on oviposition site preference in Drosophila melanogaster. Aust. J. Biol. Sci., 39, 155-160.

RUIZ-DUBREUIL, D. G. AND DEL SOLAR, E. 1991. Genetic influences on gregarious oviposition in Drosophila melanogaster. Evolución Biológica, 5, 161-171.

RUiz-Dubreuil, D. G. AND DEL SOlAR, E. 1993. A diallel analysis of gregarious oviposition in Drosophila melanogaster. Heredity, 70, 281-284.

RuiZ-Dubreuil, G., BURNET, B. AND CONNOLly, K. 1994. Behavioural correlates of selection for oviposition by Drosophila melanogaster females in a patchy environment. Heredity, 73, 103-110.

SANG, J. H. 1949. The ecological determinants of population growth in a Drosophila culture. III. Larval and pupal survival. Physiol. Zool., 22, 183-202.

SANTOS, M., FOWLER, K. AND PARTRIDGE, L 1994. Geneenvironment interaction for body size and larval density in Drosophila melanogaster: an investigation of effects on development time, thorax length and adult sex ratio. Heredity, 72, 515-521.

sChöne, H. 1984. Spatial Orientation. Princeton University Press, Princeton.

SEWELL, D., BURNET, B. AND CONNOLLY, к. 1975. Genetic analysis of larval feeding behaviour in Drosophila melanogaster. Genet. Res., 24, 163-173.

SHORROCKS, B. 1990. Coexistence in a patchy environment. In: Shorrocks, B. and Swingland, I. R. (eds) Living in a Patchy Environment, pp. 91-106. Oxford University Press, Oxford.

SOKAL, R. R. AND ROHLF, F. J. 1981. Biometry, 2nd edn. W. H. Freeman, New York.

soKolowskı, м. в. 1980. Foraging strategies of Drosophila melanogaster: a chromosomal analysis. Behav. Genet., 10, 291-302.

SOKOLOWSKI, M. B. AND HANSELL, R. 1. C. 1983. Elucidating the behavioural phenotype of Drosophila melanogaster larvae: correlations between larval foraging strategies and pupation height. Behav. Genet., 13, 267-280. 